\title{
Impaired JAK-STAT pathway signaling in leukocytes of the frail elderly
}

Leonard Daniël Samson ${ }^{1,2^{*}}$ (D) Peter Engelfriet ${ }^{1}$, W. M. Monique Verschuren ${ }^{1,3}$, H. Susan J. Picavet ${ }^{1}$, José A. Ferreira', Mary-lène de Zeeuw-Brouwer ${ }^{1}$, Anne-Marie Buisman ${ }^{1}$ and A. Mieke H. Boots ${ }^{2}$

\begin{abstract}
Background: Elderly often show reduced immune functioning and can develop chronic low-grade inflammation. Why some elderly are more prone to become frail is unknown. We investigated whether frailty is associated with altered cytokine signaling through the JAK-STAT pathway in leukocytes of 34 individuals aged 65-74 years. In addition, we investigated how this relation is affected by chronic low-grade inflammation during the previous 20 years. Cytokine signaling was quantified by measuring intracellular STAT1, STAT3, and STAT5 phosphorylation in monocytes, B cells, CD4 ${ }^{+}$T cells and $C D 8^{+}$T cells upon stimulation with IL-2, IL-6, IL-10, IFNa and IFNy, using phospho-flow cytometry. Presence of chronic low-grade inflammation was investigated by evaluating 18 different plasma inflammatory markers that had been measured repeatedly in the same individuals over the previous 20 years. Frailty was assessed as a score on a frailty index.

Results: We found that lower cytokine-induced PSTAT responsiveness in the various cell subsets was seen with higher frailty scores in both men and women, indicative of dysfunctional PSTAT responses in frailer individuals. Associations differed between men and women, with frailer women showing lower pSTAT1 responses in monocytes and frailer men showing lower pSTAT5 responses in CD4 ${ }^{+}$and $\mathrm{CD} 8^{+} \mathrm{T}$ cells. Notably, lower IL-10induced pSTAT3 responses in men were related to both higher frailty scores and higher CRP levels over the past 20 years. This might indicate poor resolution of low-grade inflammation due to defective regulatory PSTAT signaling in older men.
\end{abstract}

Conclusions: Our results emphasize the importance of preserved JAK-STAT pathway signaling in healthy aging and reveal cellular PSTAT levels as a candidate biomarker of frailty.

Keywords: Immunosenescence, Frailty, JAK-STAT pathway, Immune function, Phospho-flow cytometry, Chronic lowgrade inflammation

\footnotetext{
* Correspondence: leon.samson@rivm.nl

${ }^{1}$ National Institute of Public Health and the Environment, Bilthoven, The

Netherlands

${ }^{2}$ Department of Rheumatology and Clinical Immunology, University of

Groningen, University Medical Center Groningen, Groningen, The

Netherlands

Full list of author information is available at the end of the article
}

(c) The Author(s). 2021 Open Access This article is licensed under a Creative Commons Attribution 4.0 International License, which permits use, sharing, adaptation, distribution and reproduction in any medium or format, as long as you give appropriate credit to the original author(s) and the source, provide a link to the Creative Commons licence, and indicate if changes were made. The images or other third party material in this article are included in the article's Creative Commons licence, unless indicated otherwise in a credit line to the material. If material is not included in the article's Creative Commons licence and your intended use is not permitted by statutory regulation or exceeds the permitted use, you will need to obtain permission directly from the copyright holder. To view a copy of this licence, visit http://creativecommons.org/licenses/by/4.0/ The Creative Commons Public Domain Dedication waiver (http://creativecommons.org/publicdomain/zero/1.0/) applies to the data made available in this article, unless otherwise stated in a credit line to the data. 


\section{Introduction}

Adequate functioning of the immune system is thought to be a pivotal factor in the healthy aging process [1]. Older people tend to have less adequate immune responses to infections, including in particular respiratory infections such as COVID-19 [2]. Also vaccine responses generally are weaker $[3,4]$, as are immune functions for surveillance and clearing of (pre)malignant cells [5]. An important sign of immune dysregulation is the presence of a 'sterile' low-grade chronic inflammation, more often seen in older individuals [6-8]. It is thought that this low-grade inflammation coincides with a reduced functioning of immune cells, especially that of macrophages, leading to a poor clearance of accumulating tissue debris and senescent cells from the body [9]. Also, other major innate and adaptive immune cell subsets, such as $\mathrm{CD} 4^{+}$ and $\mathrm{CD}^{+} \mathrm{T}$ cells, and $\mathrm{B}$ cells, are essential for proper immune signaling and could thus be involved in reduced functioning of the immune system. Furthermore, senescent cells could directly contribute to chronic low-grade inflammation since they produce multiple inflammatory cytokines (known as the Senescence Associated Secretory Profile, SASP), and these cells are more abundant in older people [10].

An important factor relevant to reduced immune responses in older people could be impaired cellular cytokine signaling. Many cytokines are known to signal through the Janus kinase-Signal Transducer and Activator of Transcription proteins (JAK-STAT) pathway. In this pathway, cytokines (mainly interleukins and interferons) bind to surface receptors causing a chain reaction which ultimately leads to the intracellular phosphorylation of STAT proteins. This, in turn, can induce transcription of genes leading to adequate immune responses [11]. Loss-of-function mutations within the JAK-STAT pathway are related to life-threatening diseases such as severe combined immunodeficiency [12], and to increased susceptibility to and severity of infections $[13,14]$. Conversely, overactivation of the JAKSTAT pathway is also linked to dysfunctional immune responses. This is currently of particular interest since recently developed JAK inhibitors were shown to be effective in the treatment of auto-immune diseases such as ulcerative colitis [15] and rheumatoid arthritis [16].

JAK-STAT signaling was found to be impaired in older people, with reduced signaling [17], and higher baseline cellular STAT activation [17, 18]. Also, results of preclinical studies suggest that low-grade inflammation in older people can be reduced with JAK inhibitors $[19,20]$. The immune system in elderly may also be dysregulated due to inadequate responses to triggering of pattern-recognition receptors, such as toll-like receptors (TLR). These receptors can recognize pathogens or parts of pathogens and activate immune cells, which can in turn produce pro-inflammatory cytokines. Diminished TLR responses have been reported with higher age in most studies [21, 22] although not in a study that investigated strictly healthy older participants [23].

To better understand the role of cellular signaling in the aging process, it is important to know if reduced cellular immune responses are seen in all elderly or only in those that are frail. Frailty can be assessed by means of a frailty index score; people with a high score have been shown to have an increased risk of 'adverse' life events (e.g. injury) and a reduced capacity to recover from these events [24, 25].

In this study we investigated whether diminished immune cellular responses in older men and women are related to frailty and if impaired cytokine signaling is related to more pronounced chronic low-grade inflammation. We analyzed cytokine-induced cellular signaling through the JAK-STAT pathway and studied cellular activation through the TLR pathway in 34 individuals selected from a Dutch longitudinal population-based cohort study. The presence of chronic low-grade inflammation was evaluated by analysis of 18 different inflammatory markers in blood samples that were repeatedly taken from the same individuals at 5 year intervals over the past 20 years, between 1991 and 2017.

\section{Results \\ Study population characteristics}

The 34 participants were 65-74years old (Fig. 1), selected from the Doetinchem cohort study (DCS), a longitudinal study that started in 1987 with the participants being followed up ever since $[26,27]$. CMV seropositive individuals were excluded in order to avoid CMV infection being a confounder in the study. The median frailty index score of the participants was 0.09 , ranging from 0 ( $0 / 36$ deficits present) to 0.4 (14/36 deficits) (Table 1). The mean BMI level was $26.9 \mathrm{~kg} \mathrm{~m}^{-2}$ and ranged from $20.8-35 \mathrm{~kg} \mathrm{~m}^{-2}$. Frailty index scores (see Table S1 for frailty index components), BMI levels and age did not differ significantly between men and women (Fig. S1A-C).

\section{Cytokine-induced pSTAT responsiveness within different immune cell lineages}

We used a broad panel of experimental conditions to quantify immune cell cytokine responses by separately stimulating PBMCs with IL-10, IFN $\gamma$, IL-6, IL-2, or IFN $\alpha$, and subsequently measuring phosphorylated STAT1, STAT3, and STAT5 (pSTATs) in monocytes, $\mathrm{B}$ cells, and $\mathrm{CD}^{+}$and $\mathrm{CD}^{+} \mathrm{T}$ cells (Fig. 1), using phosho-flow cytometry (Fig. S2). Examples of individual pSTAT expression patterns after stimulation can be found in Fig. S3. Baseline (unstimulated) levels of pSTAT1, pSTAT3, and pSTAT5 were higher in 


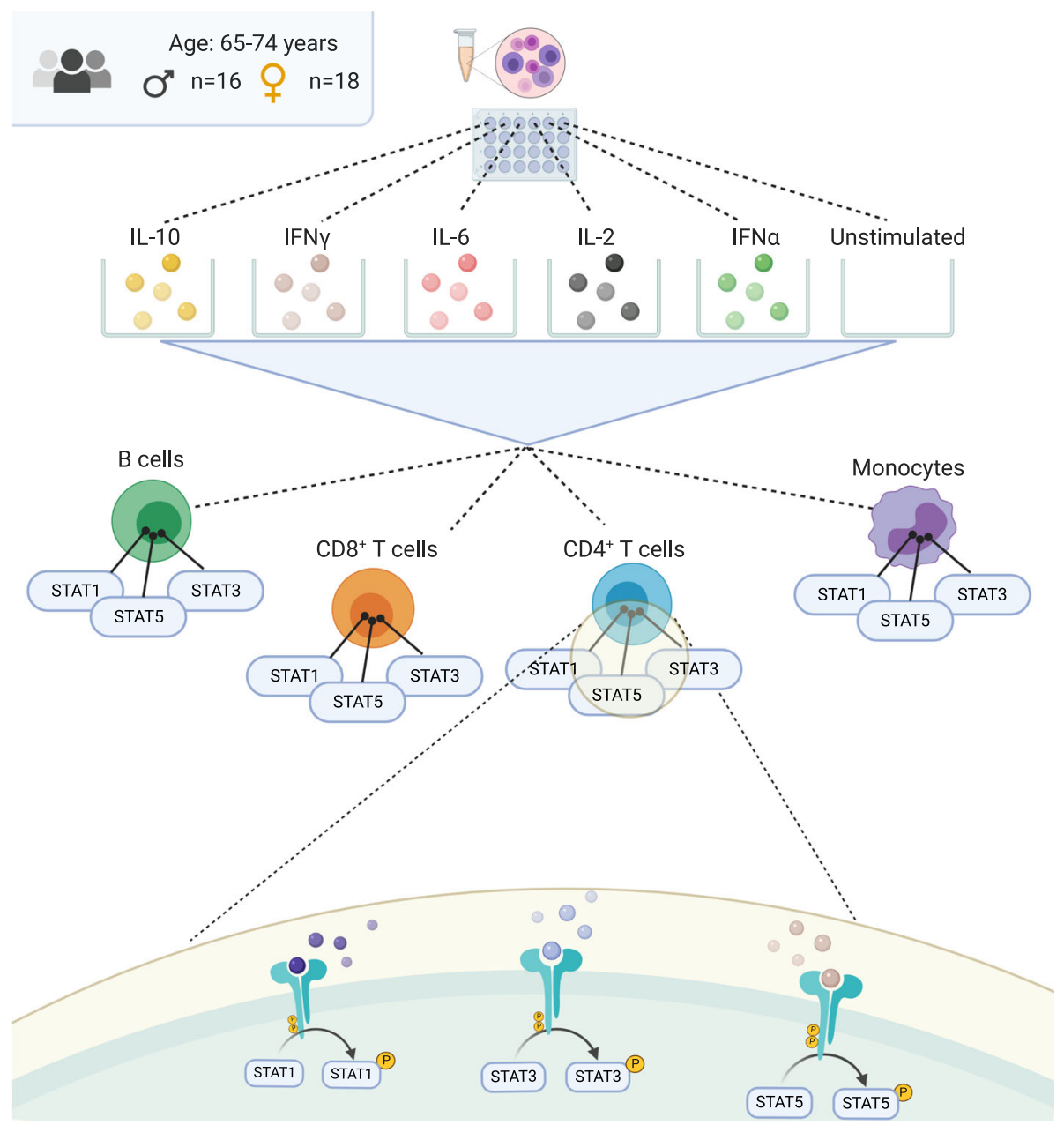

Fig. 1 Study and experimental design. CMV-seronegative participants were selected from the Doetinchem cohort study. PBMCs were thawed and stimulated with the indicated cytokines or were left unstimulated (baseline). Intranuclear immunofluorescence staining was performed to quantify phosphorylation of STAT1, 3, and 5 proteins, in combination with surface staining for lineage-specific markers to distinguish between B cells, monocytes, and T cells (CD8 ${ }^{+}$and $\left.\mathrm{CD} 4^{+}\right)$

monocytes than in the other cell subsets, and those of pSTAT5 were higher than those of pSTAT1 and pSTAT3 in both $\mathrm{CD}^{+}$and $\mathrm{CD}^{+} \mathrm{T}$ cells. (Fig. S4, light grey density plots). The baseline pSTAT levels did not differ significantly between men and women (Table S2). Monocytes showed relatively high induced pSTAT levels (after stimulation), besides high baseline pSTAT levels. As expected, not all experimental conditions induced responses of pSTAT1, pSTAT3 and pSTAT5; therefore we selected the conditions showing a 'clear' response upon stimulation (defined as a median fold change $\geq 2$ ) for further analysis (Fig. 2, Fig. S5, 20 out of 60 stimulation conditions). Most of such responses were induced by IFN $\alpha$, which is indeed expected to induce not only phosphorylation of STAT1, but also STAT3 and pSTAT5 through the type I IFN receptor [17, 28, 29]. Other responses described in literature that we observed were IL2STAT5 responses in $\mathrm{T}$ cells [12], IFN $\gamma$-STAT1 responses in monocytes and B cells [12], and IL6- and IL10- induced STAT3 responses in monocytes and $\mathrm{CD}^{+} \mathrm{T}$ cells. IL6 also induced STAT1 in CD4 ${ }^{+} \mathrm{T}$ cells, which has been shown before [30]. The highest responses were seen in $\mathrm{CD}^{+}$and $\mathrm{CD} 8^{+} \mathrm{T}$ cells after stimulation with IL-10, with a fold change in pSTAT3 levels of 11.9 and 15.2, respectively (Table S3). When testing for sex-specific differences, we did not observe differences in baseline pSTAT levels between men and women, but we did find that women had higher pSTAT3 responses to IL-10 stimulation in $\mathrm{CD}^{+} \mathrm{T}$ cells and to some extent also in $\mathrm{CD}^{+} \mathrm{T}$ cells, although the differences were small (Table S4, Fig. 2). Next, we examined how cell subset numbers differed between the sexes and with frailty in this population, 
Table 1 Baseline characteristics of the study population $(n=34)$

\begin{tabular}{ll}
\hline$\%(\mathrm{n})$ of women & 52.9 (18) \\
Age, yrs. & 69.4 (SD: 2.6, range: 65.4-74.1) \\
BMl, $\mathrm{kg} \mathrm{m}^{-2}$ & 26.9 (SD: 3.4, range: 20.8-35) \\
Frailty index score & 0.09 (0.18, range: 0-0.4) \\
CMV-, \% & 100
\end{tabular}

Frailty index score: health score (median + interquartile range) enumerating 36 health deficits, theoretically ranging from 0 (no deficits) to 1 (all deficits). BMI and age are in mean + standard deviation. CMV-seronegative study participants were selected for this study. \% of women: percentage of women of the total study population

since a difference in cell numbers might be a confounder in our analyses. We observed that women on average had higher numbers of $\mathrm{B}$ cells but lower numbers of monocytes than men (Fig. S6A). In line with our previous data [31], monocyte numbers in women, but not men, were higher with higher frailty index scores (Fig. S6B).

\section{Frailty is associated with lower cellular pSTAT} responsiveness

We related frailty score to baseline pSTAT levels and found a negative association between a higher frailty score and baseline pSTAT1 in monocytes of men but not women (Fig. 3A and B). When we investigated the relationship of frailty with the cellular pSTAT cytokine responsiveness (upon cytokine stimulation), most of the observed associations were negative, i.e. negative correlations, and differed between men and women (Fig. 4A).

Associations of pSTAT1 responsiveness with frailty were mainly seen in women (Fig. 4A, B). pSTAT1 responses of monocytes (Fig. 4B, $\rho=-0.53$ after IFN $\alpha$ and $\rho=-0.52$ after IFN $\gamma$ stimulation) and of $\mathrm{CD}^{+}{ }^{+} \mathrm{T}$ cells (Fig. 4B, $\rho=-0.64$ after IL-6 stimulation) were found to be weaker in women with a higher frailty index score ('frailer' women). In contrast, pSTAT1 responses of monocytes were found to be stronger in frailer men

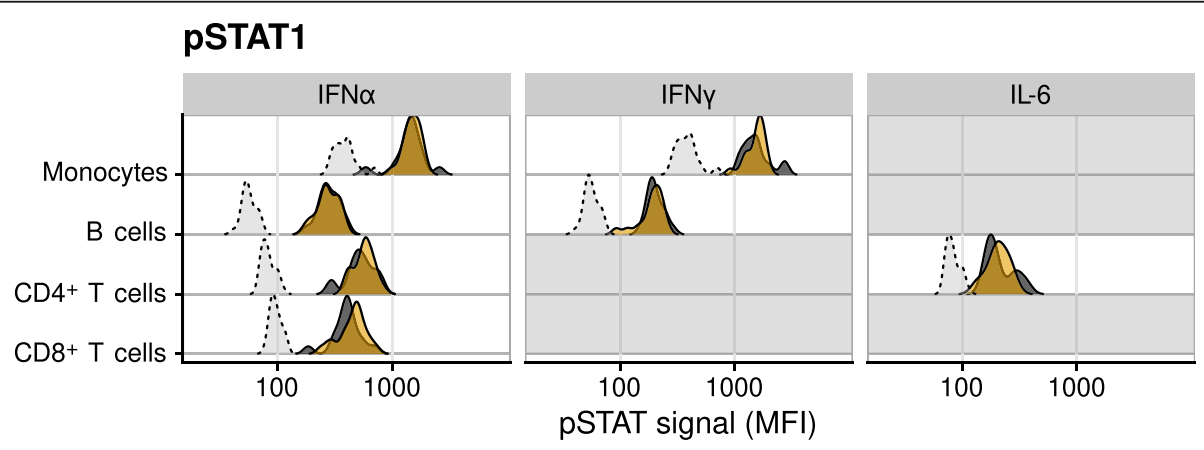

\section{pSTAT3}
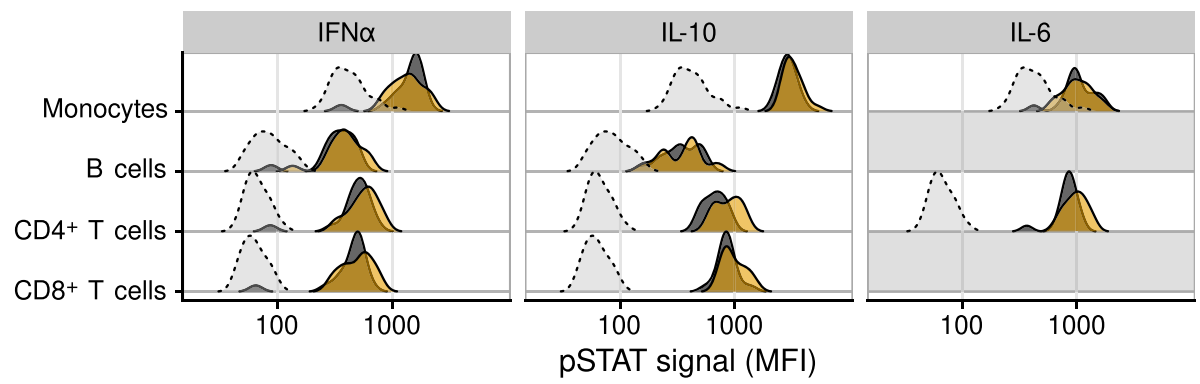

\section{pSTAT5}

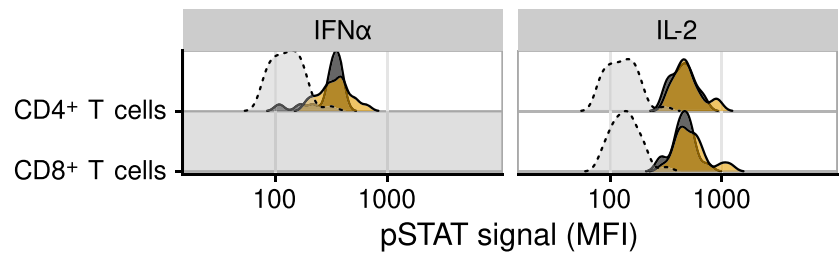

Men

Women

Fig. 2 Levels of phosphorylated STAT proteins (pSTAT1, pSTAT3, and pSTAT5), measured in CD4 ${ }^{+} \mathrm{T}_{\text {cells, }}$ CD8 ${ }^{+} \mathrm{T}$ cells, B cells, and monocytes. Grey surface area with dashed outline shows baseline (unstimulated) pSTAT levels. Dark grey and orange surface areas with solid outline show PSTAT immune cell responses to stimulation in men and women, respectively. Shown are 20 out of 60 experimental stimuli conditions that induced a robust change (fold change $>2$ ) compared to the baseline condition in $\mathrm{CD} 4^{+}, \mathrm{CD} 8^{+}$, B cells and monocytes 
$(\rho=0.60)$ after IFN $\gamma$ stimulation. Negative associations of pSTAT3 responsiveness with frailty were seen in B cells of men and $\mathrm{T}$ cells of both men and women, with most associations observed in men (Fig. 4A, C). IFN $\alpha$ induced pSTAT3 responses were negatively related to frailty in $\mathrm{CD}_{4}^{+} \mathrm{T}$ cells of women but not in men, and to $\mathrm{CD}^{+} \mathrm{T}$ cells of both men and women. In men, other negative relationships were found between IL-10induced pSTAT3 responses and frailty in B cells, CD4 ${ }^{+}$ $\mathrm{T}$ cells, and $\mathrm{CD}^{+} \mathrm{T}$ cells $(\rho=-0.65, \rho=-0.59, \rho=-$ 0.66 , respectively). Lastly, associations of pSTAT5 responsiveness with frailty were only seen in $\mathrm{T}$ cells of men (Fig. 4D). Negative associations in men were found of IFN $\alpha$-induced pSTAT5 responses with frailty in $\mathrm{CD}_{4}^{+}$ $\mathrm{T}$ cells $(\rho=-0.69)$ and of IL- 2 induced pSTAT5 responses with frailty in $\mathrm{CD}^{+}$and $\mathrm{CD}^{+} \mathrm{T}$ cells $(\rho=-$ 0.71 and -0.82 , respectively).

It is known that BMI can influence the inflammatory profile and that BMI is generally higher in frail individuals. When associations were directly tested between BMI and the cellular pSTAT responses, they had similar direction as with frailty, but none of the associations was strong enough with an acceptable false discovery rate (Fig. S7).

Thus, in general multiple associations of pSTAT responsiveness with frailty were seen, with overall lower cytokine induced cellular responsivity in frailer individuals. The direction of the associations was mostly similar in men and women. However, the strength of the associations differed, with more associations found in men. Furthermore, impaired pSTAT1 responsiveness of monocytes was found in frailer women and impaired pSTAT5 responsiveness of $\mathrm{CD}^{+}$and $\mathrm{CD}^{+} \mathrm{T}$ cells in frailer men.

Defective regulatory pSTAT signaling in men with chronic low-grade inflammation

Since chronic low grade inflammation may impact cellular responsiveness, we next explored whether cellular
pSTAT levels were related to any of 18 different inflammatory markers that were measured longitudinally, namely with 5 -year intervals over the past 20 years in the same individuals. Cumulative exposure to low-grade inflammation over this time period was estimated, by calculating the area under the curve of every inflammatory marker for each individual. We then related the AUC's to their cellular pSTAT responses. A clustering algorithm identified three clusters of markers based on Spearman's $\rho$ values (Fig. 5A). As the heatmap shows, positive correlations were more prominent in women, which contrasted with the more prominent negative ones in men, especially in clusters 2 and 3. Higher CRP levels in the past 20 years in men were associated with lower IL-10 induced pSTAT3 responses (Fig. 5A, B), which might indicate defective regulatory IL-10 signaling in men. This association was the one with the highest $\rho$ value $(\rho=-0.85)$ and the only one below the pre-set false discovery rate threshold. Other high $\rho$ values $(\rho>$ 0.7 or $\rho<-0.7)$ were also seen between CRP levels and IL-10 induced pSTAT3 responses in B cells in men ( $\rho=$ - 0.71) (Fig. 5B). In women the highest $\rho$ value was found for the relationship between sGP130 levels and IL-6 induced pSTAT3 CD4 ${ }^{+} \mathrm{T}$ cell responses $(\rho=0.72)$. Of note is that, while frailty was related to several pSTAT responses induced by IL-10 and IL-6, no association was found of pSTAT responsiveness with IL-10 or IL-6 levels in the circulation. Thus, only in men associations were found between pro-inflammatory marker levels in the past 20 years and pSTAT responses, possibly showing signs of a defective IL-10 signaling.

\section{No associations found between frailty and immune cell cytokine production after TLR stimulation}

We also investigated if cytokine production is different in frail individuals after activation of specific pattern recognition receptors that are thought to be involved in
A

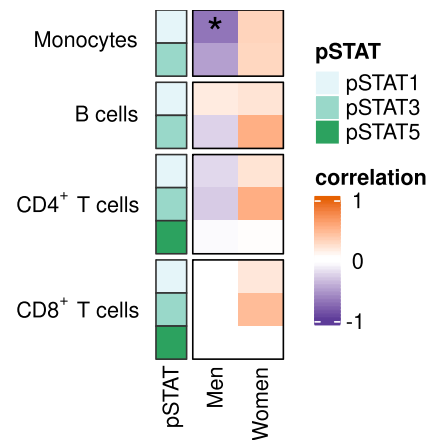

B Baseline pSTAT1 in monocytes

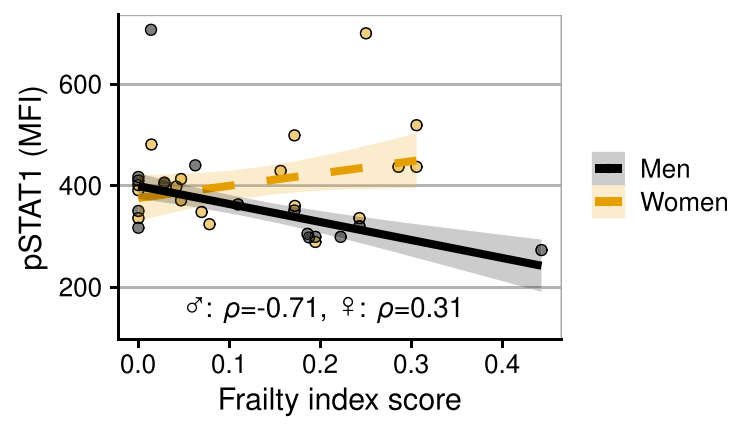

Fig. 3 A Heatmap showing correlation coefficients of the relation between frailty and the baseline (unstimulated) phosphorylation of STAT1, STAT3, and STAT5 in monocytes, B cells, and CD4 ${ }^{+}$and CD $8^{+} T$ cells. Every box displays the Spearman's $\rho$ value based on data of 16 men and 18 women. B Baseline (unstimulated) pSTAT1 expression in monocytes. Solid trendline in (B) indicates that an association was found 


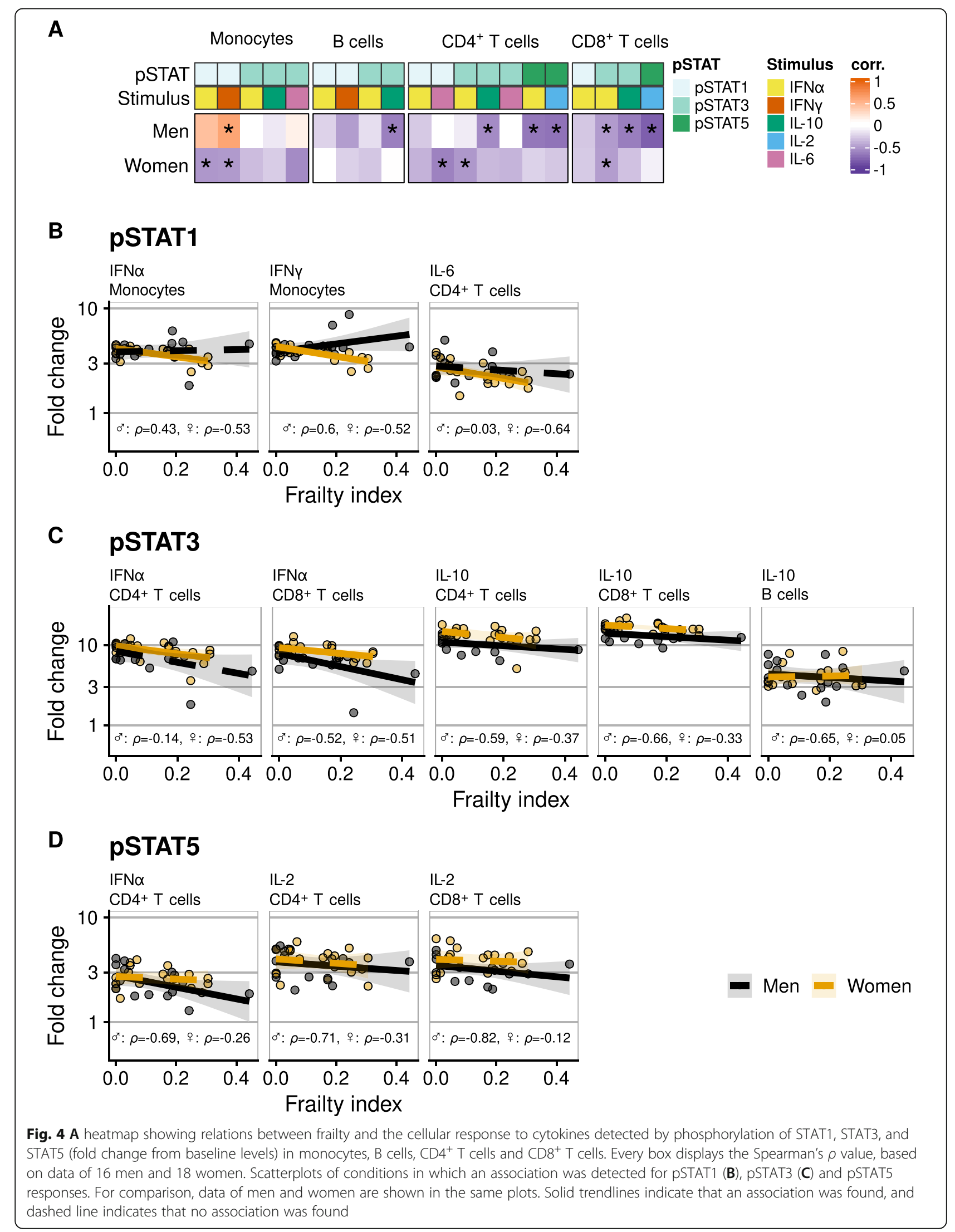




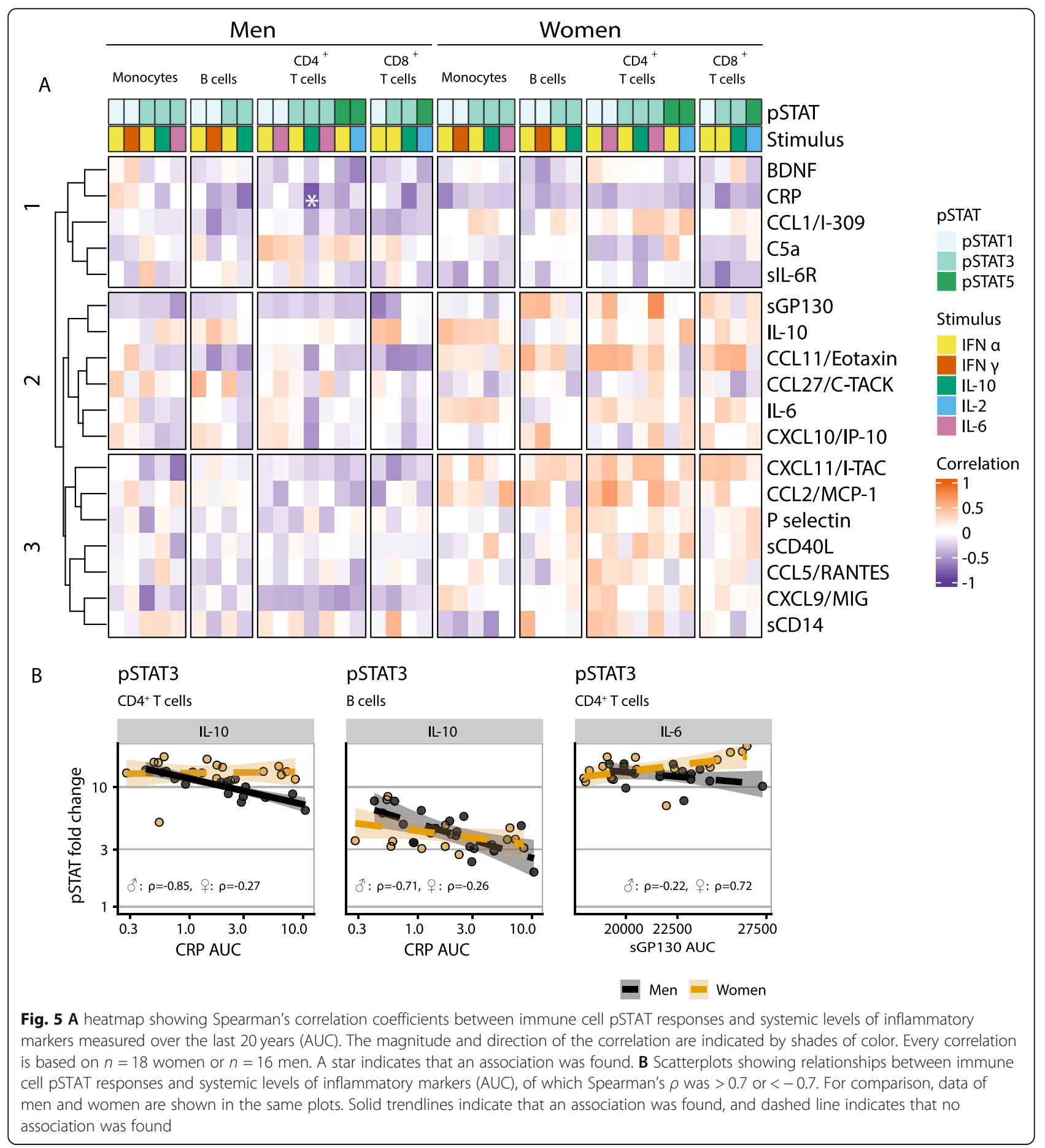

inflammation. After PBMCs were stimulated for $24 \mathrm{~h}$ with agonists of either Toll-like receptor (TLR)4 (lipopolysaccharide, LPS), TLR7/8 (Resiquimod, R848) or TLR9 (CPG ODN), we measured production of the cytokines IFN $\alpha$, IFN $\gamma$, TNF $\alpha$, IL-10, IL-1 $\beta$, IL-8, MCP-1, CXCL10 and of sGP130. Stimulation with LPS and R848 resulted in enhanced cytokine production for most cytokines, while CpG ODN stimulation generally resulted in no or low cytokine production. Levels of IFN $\alpha$ were below detection limit after $24 \mathrm{~h}$ stimulation for the majority of the samples. A selection was made of 20 'clear' responses to TLR-ligands ('clear' defined as median $>2$ fold change in cytokine production compared to control sample), which were used for further analyses (Fig. 6A, B). Cytokine production after TLR activation of PBMCs did not differ between men and women when testing 
either cytokine concentrations after stimulation (Fig. 6A) or fold change in concentration (Fig. 6B, Table S9). Regarding correlations between frailty and PBMC cytokine production conditions, more negative Spearman's $\rho$ values were found in men than in women, but no association with frailty was found in either men or women (Fig. 6C, Table S10).

\section{Discussion}

Our main finding is that frailty in older individuals of 65-74 years coincides with lower cellular pSTAT responsiveness. Negative associations between pSTAT responsiveness and frailty were found in both men and women. More and stronger associations were found in men, with signs of lower immune regulatory (IL-10 induced) pSTAT3 responses in frailer men. These lower responses in frailer men were related to higher levels of CRP in the past 20 years. Our results extend previous knowledge that showed lower pSTAT responses at higher age and in cardiovascular dysfunction [17], and underline the importance of the JAK-STAT pathway in frailty and, possibly, immunosenescence.

To our knowledge, no other studies related cellular pSTAT responsiveness to frailty. Lower pSTAT responses were previously found to be related to markers of cardiovascular disease and to chronological age [17]. More specifically, IFN $\alpha$-induced pSTAT3 in $\mathrm{CD}^{+} \mathrm{T}$ cells and pSTAT5 in $\mathrm{CD} 4^{+} \mathrm{T}$ cells were negatively associated with age. Our data extend these findings by showing that IFN $\alpha$-induced pSTAT3 responses of $\mathrm{CD}^{+} \mathrm{T}$ cells were also negatively associated with frailty in both men and women and that IFN $\alpha$-induced pSTAT5 responses in $\mathrm{CD}^{+} \mathrm{T}$ cells were negatively associated with frailty in older men. While previous studies found higher levels of baseline pSTAT levels at higher ages $[17,18]$ and with higher CRP levels [17], we found an opposite relationship with frailty instead, namely lower baseline pSTAT1 levels in monocytes of frailer men. Given these previous findings, this was unexpected since chronic low-grade inflammation is seen more often in frail individuals [32-34]. Thus, it is unclear how this should be interpreted with regard to the theory that immune cells of frail older people show higher baseline JAK-STAT pathway activation. We speculate that baseline STAT phosphorylation is reduced in immune cells of frail elderly due to these cells being continuously triggered by chronic low-grade inflammation, leading to cellular exhaustion; further studies are needed to confirm this hypothesis. On the other hand, impaired cytokine signaling may cause reduced immune responses, leading to accumulation of damage-associated molecular patterns and thereby induction of chronic low-grade inflammation. Thus, while it is unclear whether chronic low-grade inflammation is a cause or a consequence of impaired cytokine signaling, both can enhance each other, are part of a dysfunctional immune system and probably lead to reduced immune responses that are clinically meaningful, due to their associations with frailty.

Of interest is the association we found between higher CRP levels in the previous 20 years, a sign of chronic low grade inflammation, and lower IL-10 induced pSTAT3 responses in men. Since we also observed lower pSTAT3 responsiveness to IL-10 in lymphocytes of frailer men, this might suggest that their lymphocytes are less responsive to anti-inflammatory signals and therefore less able to control inflammation. These findings are in agreement with results of previous studies showing that chronically elevated levels of CRP are related to frailty [32-34]. Recent studies noted that severely ill COVID-19 patients show elevated levels of IL-10 [35] which led some investigators to propose that IL-10 might contribute to the severity of the disease, because IL-10 is known to have both pro- and anti-inflammatory properties [36]. Given that the risk of developing severe symptoms in COVID-19 is greater in older men than in older women [37], and since we showed signs of defective IL-10 signaling in frail men, we speculate that defective downstream IL-10 -STAT3 signaling contributes to the severity of infectious diseases such as COVID-19 due to the reduced immune regulatory function of IL10. Indeed, JAK-STAT pathway signaling has been shown to be pivotal in developing severe symptoms such as a cytokine release syndrome in COVID-19 patients [38], in particular through STAT3 signaling [39].

Many clinical trials are currently ongoing to investigate efficacy of blocking JAK-STAT signaling with JAK inhibitors in the treatment of COVID-19 [38]. While phase II studies showed promising results [40, 41], early reports from phase III trials are mixed, with some positive [42] and some negative results [43]. These mixed results might be explained by different timing of drug administration, and by improper identification of the patients who benefit most from JAK/STAT inhibition [38]. This should be addressed in future studies which should also elucidate whether impaired regulatory IL-10 STAT3 signaling can be a marker of developing severe reactions to infectious diseases. This would strengthen the rationale for targeting this pathway. Thus, cellular pSTAT levels may qualify as a biomarker to help to identify patients that benefit most from JAK inhibitor treatment.

An important factor in the association between frailty and reduced cytokine responsiveness could be overweight, since overweight has been associated with impaired JAK-STAT responses in adipocytes [44]. We were unable to adjust the results for BMI directly due to the small sample size. However, we did not find associations of pSTAT responses with BMI, which may imply that BMI is not the main driver in the association of 


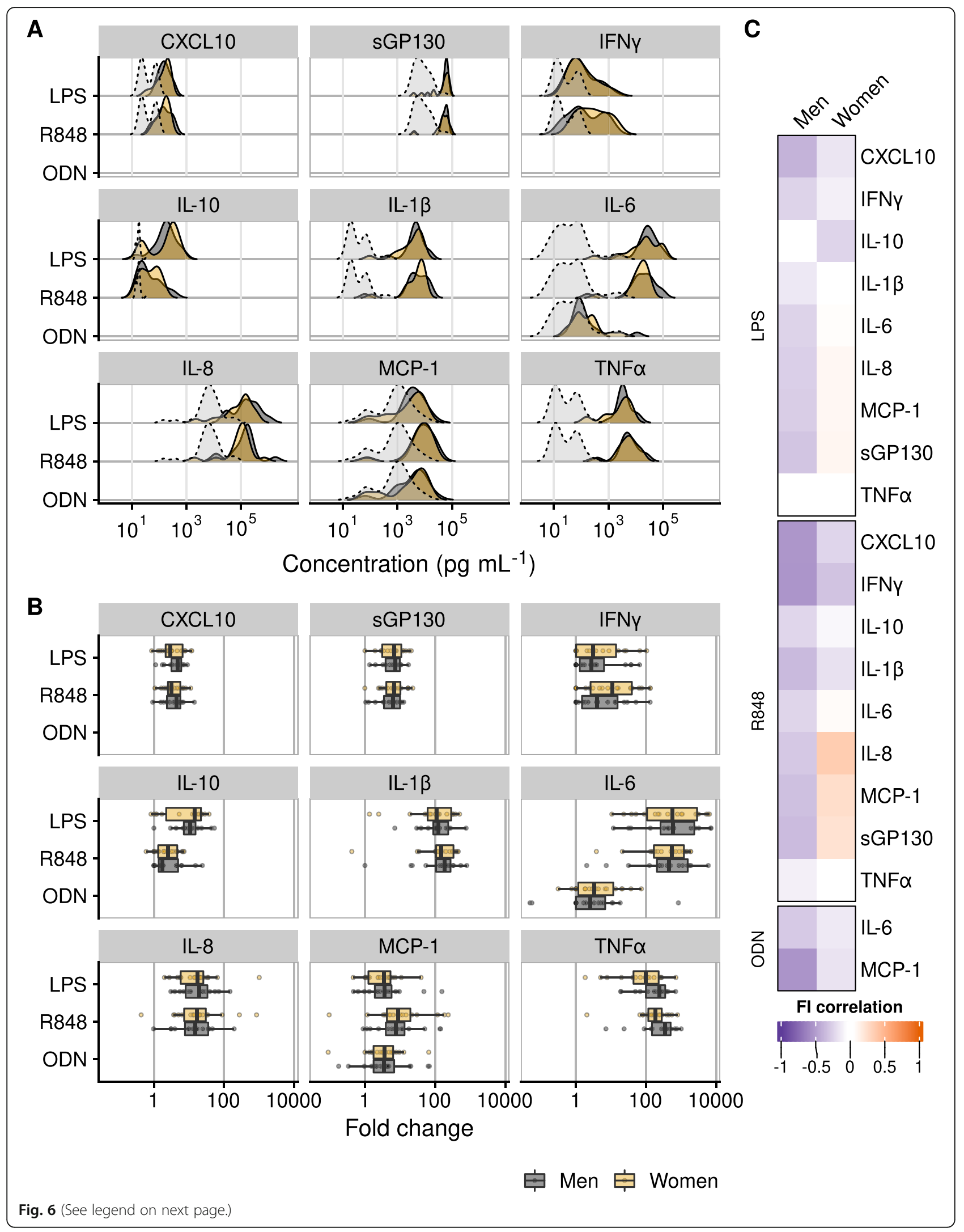


(See figure on previous page.)

Fig. 6 A Density plots of cytokine production by PBMCs after $24 \mathrm{~h}$ stimulation with medium (light grey density, spontaneous cytokine production) or with R848, ODN, or LPS. B Cytokine production by PBMCs upon 24 h stimulation with TLR4 (LPS), TLR7/8 (R848), and TLR9 (CpG ODN) agonists. Plots are shown only for cytokines with a fold change $>2$. C Heatmap showing Spearman's $\rho$ values of correlations between frailty and PBMC cytokine production

pSTAT1, pSTAT3, and pSTAT5 responses in immune cells and frailty.

While we found that cytokine responsiveness is lower in frail people, we did not find an association of frailty with cellular cytokine production after stimulation with TLR agonists such as LPS, R848 and CpG ODN. This might mean that the initial intracellular reaction to pattern associated molecular patterns is still intact, but that the response to TLR-induced cytokines is reduced in frail individuals due to reduced JAK-STAT pathway signaling. Results should be interpreted with care, as the high dosage of the TLR agonists chosen for in vitro stimulation may overcome or mask possible subtle invivo differences in TLR responses.

To the best of our knowledge, this is the first study describing differences in pSTAT responses of leukocytes in men and women. Lower cellular pSTAT responsiveness in frailer participants was seen in both men and women, with the direction of the correlations being the same in men and women for most of the experimental conditions. However, impaired signaling in frailer participants was seen with different pSTATs in men and women, and in general the observed associations were stronger in men. Of interest is that pSTAT1 cytokine signaling was found to be more impaired in frail women than in men, while frail women were also found to have higher monocyte numbers. This is consistent with impaired phagocyte functioning in frail women, with more monocytes being produced due to their impaired cellular signaling capacity as a possible compensatory mechanism. On the other hand, pSTAT5 signaling was found to be impaired in frail men but not in frail women. It is known that pSTAT5 responses can be initiated by growth hormones [45]. Growth hormone secretion is higher in women than in men and is influenced by sex hormones such as estrogens [45], which might explain why we found the associations between pSTAT5 responses and frailty in men but not in women. Sex hormone levels might also further help to explain the differences in pSTAT responsiveness that we found between men and women, since STATs are known to interact with the androgen receptor [46] and pSTAT activation is influenced by estrogen and progesterone levels $[47,48]$.

Strengths of the study are the in-depth pSTAT signaling assay that we used, which allowed us to characterize a wide range of functional cellular cytokine response signals. Another advantage is that we could build on the extensive information and biomaterials gathered in the course of a unique longitudinal cohort study. This gave us the opportunity to compile and score a comprehensive frailty index in older individuals and to measure inflammatory markers longitudinally over a period of 20 years to quantify chronic low-grade inflammation. Our study also comes with limitations. First, our sample size was relatively low, so results cannot be extrapolated to the general population. Furthermore, the cellular pSTAT responsiveness could not accurately predict frailty by a prediction model due to the small sample size and the relatively small changes in effect size (data not shown). Future studies with larger populations, preferably including participants that are young and healthy both with and without low-grade inflammation, could help to establish clinical usefulness of our results and to determine whether defective signaling precedes frailty or not. In addition, it would be useful to measure levels of reproductive hormones to evaluate their influence on reduced pSTAT signaling in frail individuals. Another noteworthy point is that, while we used a large panel of cytokines signaling via JAK/STAT pathways we did not study activation of other known STATs, namely STAT2, STAT4 and STAT6. Larger phospho-flow panels may give a more complete description of cellular responsiveness in frail older people. Also, expansion of the staining panels with, for example, phenotypic markers to improve $\mathrm{CD}^{+} \mathrm{T}$ cell gating, could also be considered in future studies. Furthermore, other innate signaling cytokines such as IL-4 and IL- 8 could be considered to be included, especially since the function of myeloid lineage cells is thought to become impaired with age $[5,49]$ and numbers of myeloid cells are higher in frail older people [31]. Lastly, how the results translate to invivo signaling remains to be investigated. In-vivo signaling might differ from in-vitro signaling, as the context is different and in vivo cytokine receptor expression might vary due to e.g. the presence of specific antigen, co-stimulation, or a difference in strength and duration of stimulation.

\section{Conclusion}

In summary, our study gives important insights into relationships between immune functioning and frailty, and revealed sex-specific differences. We found cellular pSTAT responses to be reduced in older frail individuals. Interestingly, frail men show signs of defective regulatory pSTAT cytokine signaling and this is associated with chronic low-grade inflammation in the past 20 years. The data imply that the JAK/STAT signaling pathway is important in the aging process and therefore 
markers of this pathway could have utility as biomarkers of frailty. This is of particular interest since multiple drugs have been developed that can target the JAK/ STAT pathway. We hope that these data encourage further research investigating how impaired JAK-STAT signaling is related to a poor immunological response to vaccines and infections and to chronic low-grade inflammation in older individuals.

\section{Methods}

The study participants take part in the ongoing Doetinchem cohort study (DCS) [26, 27]. In the DCS, six consecutive measurement and sampling rounds, every 5 years, have been completed (1993-1997, 1998-2002, 2003-2007, 2008-2012, 2013-2017), while the seventh round is still ongoing (2018-2022). During each measurement round, plasma samples were taken and information regarding the participants' health was collected. The present analysis is based on a sample of 16 men and 18 women who were 65-74 years of age, selected from a subgroup of DCS participants in which we previously analyzed inflammatory marker trajectories [50]. The latter were still active in the DCS at least until 2016, had at least 5 plasma samples available from previous rounds, were CMV seronegative at last measurement, and did not use immunosuppressants. From among those, we chose individuals with the highest and with the lowest frailty index, in equal numbers. The sample size was restricted by budgetary and logistic constraints.

\section{Frailty index}

Frailty of participants was evaluated with a frailty index score. Details on the frailty index score used in this study can be found elsewhere [33]. In short, the frailty index score was based on previous studies $[24,25,51$, 52] and consists of 36 health deficits assessed by questionnaires and objective measurements. Examples are cognitive deficits obtained from validated cognitive tests, physical deficits such as poor handgrip strength, psychological deficits, and deficits regarding living independently (Table S2). The values that the frailty index can take are restricted between 0 (best possible score) and one (worst possible score). The frailty index was calculated based on data of the last two DCS assessment rounds (round 5 and 6) and was validated in the Doetinchem cohort study [33].

\section{PBMC isolation}

Peripheral blood mononuclear cells (PBMCs) were isolated from heparinized blood by Lymphoprep (Progen) density gradient centrifugation, according to the manufacturer's instructions. After isolation, the cells were washed with PBS (Gibco) containing 0.2\% FCS, and then frozen in a solution with $90 \%$ fetal calf serum and $10 \%$ dimethyl sulfoxide at $-135^{\circ} \mathrm{C}$ until further use.

\section{PBMC cytokine response analysis using phospho-flow cytometry}

PBMCs from all participants $(n=34)$ were stimulated with cytokines to measure JAK-STAT pathway activation by quantifying the phosphorylation of STAT 1,3 and 5 (Fig. 1). We used a barcoding technique as described previously $[17,53]$. PBMCs were rapidly thawed and washed in RPMI (Thermofisher Scientific) with 10\% fetal bovine serum. After $1 \mathrm{~h}$ resting, the PBMCs were stimulated with one cytokine per well (deep well plates, Sigma-Aldrich, $0.5^{*} 10^{6}$ PBMCs per well) for $30 \mathrm{~min}$ at $37^{\circ} \mathrm{C}, 5 \% \mathrm{CO}_{2}$ with the cytokines IL-2 (R\&D Systems, $50 \mathrm{ng} \mathrm{mL}^{-1}$ ), IL-6 (R\&D Systems, $25 \mathrm{ng} \mathrm{mL}^{-1}$ ), IL-10 (Peprotech, $100 \mathrm{ng} \mathrm{mL}^{-1}$ ), IFNa (R\&D Systems, $0.5^{*} 10^{4}$ $\mathrm{U} \mathrm{mL}^{-1}$ ) and IFNY (Peprotech, $50 \mathrm{ng} \mathrm{mL}^{-1}$ ). One well was reserved as the control condition, without stimulus. Next, PBMCs were fixated (in the deep well plate) with 1.6\% paraformaldehyde (Alfa Aesar) for $10 \mathrm{~min}$ at room temperature, and thereafter permeabilizated with icecold $\left(-20^{\circ} \mathrm{C}\right) 100 \%$ methanol for $5 \mathrm{~min}$, at $4{ }^{\circ} \mathrm{C}$. After permeabilization, the methanol was diluted 1:1 with cold PBS and the individual samples were stained for $30 \mathrm{~min}$, $4{ }^{\circ} \mathrm{C}$ with a combination of Pacific Orange succinimidyl esters $\left(0,0.13\right.$, or $\left.1 \mu \mathrm{g} \mathrm{mL}^{-1}\right)$ and Alexa Fluor 750 succinimidyl esters $\left(0,0.5\right.$, or $2 \mu \mathrm{g} \mathrm{mL}^{-1}$ Fisher Scientific), to obtain a unique bar code per condition. After barcoding, the PBMCs were extensively washed with $\mathrm{PBS} / 0,5 \%$ $\mathrm{BSA} / 2 \mathrm{mM}$ EDTA and the PBMCs from the same individual were collected and pooled in one FACS tube (BD Falcon). Thereafter, the PBMCs were stained for $30 \mathrm{~min}$ at $4{ }^{\circ} \mathrm{C}$ with $\mathrm{CD} 3(\mathrm{UCHT} 1)$-Pacific Blue, CD4(SK3)BUV395, CD20(H1)-PerCPCy5.5, and CD33(P67-6)PECy7 to distinguish $\mathrm{CD} 4^{+}$and $\mathrm{CD} 8^{+} \mathrm{T}$ cells, B cells, monocytes, respectively and with the markers STAT1(4a)-Alexa Fluor 488, STAT3(4/P)-Alexa Fluor 647 and STAT5(pY694)-PE to quantify phosphorylation of STATs (Fig. S2). Samples were measured on a flow cytometer (LSR II Fortessa X20, BD Bioscience).

\section{TLR stimulation of PBMC and cytokine production assay}

PBMCs of all participants were thawed, washed and put to rest for $1 \mathrm{~h}$ in RPMI+glutamax with $10 \%$ FBS in a 96wells round-bottom plate (Greiner), at a concentration of 200.000 PBMCs/well. After resting, the PBMCs were stimulated with one stimulus per well for $24 \mathrm{~h}$ at $37^{\circ} \mathrm{C}$, $5 \% \mathrm{CO}_{2}$. Stimuli used were LPS EK (E.coli K12 ultrapure) $10 \mathrm{ng} \mathrm{mL}^{-1}$ (TLR4), CpG ODNM362 $10 \mu \mathrm{g} \mathrm{mL}^{-1}$ (TLR9), and R848 $10 \mu \mathrm{g} \mathrm{mL}^{-1}$ (all Invivogen) (TLR7\&8). After stimulation, the supernatants were collected and stored at $-80{ }^{\circ} \mathrm{C}$ for later use. The supernatants were thawed and analyzed in two batches on a FACSCanto ${ }^{\text {ty }}$ 
flow cytometer (BD Biosciences), using a custom-made bead-based immunoassay (LEGENDplex ${ }^{\mathrm{mm}}$, BioLegend) according to the manufacturer's instructions, for the following cytokines and chemokines: sGP130, IL-6, IFNY, IL-10, IL-1 $\beta$, TNF $\alpha$, MCP-1, RANTES, CXCL8/IL-8, CXCL10/IP10, and IFN $\alpha$. In addition, concentrations of sIL6R were quantified using a commercially available ELISA kit (R\&D Systems).

\section{Plasma inflammatory protein trajectories}

To quantify low-grade inflammation, we measured a panel of inflammatory markers in 5 repeated blood samples per individual that were withdrawn at 5 -year intervals over the period of approximately 20 years; details are described elsewhere [50]. The panel consists of the following cytokines, chemokines and soluble receptors: C-C Motif Chemokine Ligand (CCL) 1/I-309, CCL2/ MCP-1, CCL5/RANTES, CCL11/Eotaxin, CCL27/CTACK, C-X-C Motif Chemokine Ligand (CXCL) 9 /MIG, CXCL10/IP-10, CXCL11/I-TAC, IL-10, IL-6, soluble CD40 ligand (sCD40L), soluble CD14 (sCD14), soluble IL-6 receptor (sIL-6R), glycoprotein 130 (GP130), Complement 5a (C5a), Brain-derived neurotrophic factor (BDNF), and soluble P-selectin. Plasma levels were measured in a multiplex immunoassay (Luminex core facility lab, UMC medical center, Utrecht, The Netherlands). Samples were stored at $-80^{\circ} \mathrm{C}$ and only thawed on the morning of the measurement, with all samples from the same participant measured on the same plate. Levels of C-reactive protein (CRP) had been measured earlier in separate plasma samples and in a separate assay [54].

\section{Statistical analysis}

\section{Association studies}

We used the permutation version of the Spearman's test or, when appropriate, Wilcoxon's test as implemented in the coin $\mathrm{R}$ package [55] to test associations for all our research questions. These association studies were performed separately for men and women. Associations were adjusted for possible confounding by batch effects. For all these association studies, we accounted for multiple testing by controlling the false discovery rate [56]. A cutoff of the false discovery rate of $15 \%$ was chosen based on the exploratory nature of this research. This means that, in theory, of all findings reported here at most $15 \%$ could be false positives. All details regarding the outcomes of the association studies, including $p$-values, are shown in supplementary information (Tables S2, S4, S5, S6, S7, S8, S9, and S10).

All analyses were performed in $\mathrm{R}$ (version 3.6.2) [57]. Several packages were used for general data wrangling [58, 59], data visualization [60-62], and customizing tables $[63,64]$. Clusters of inflammatory markers within a heatmap were defined with a hierarchical cluster algorithm using the complete linkage criterion. The clustering was performed on the dissimilarity matrix which was obtained from data of the $\rho$ values.

\section{Supplementary Information}

The online version contains supplementary material available at https://doi. org/10.1186/s12979-021-00261-w.

Additional file 1.

\section{Acknowledgments}

We would like to thank the DCS respondents for their participation in the study and the epidemiologists and fieldworkers of the Municipal Health Service in Doetinchem for their contribution to the data collection. In addition, we would like to thank Petra Vissink for her help with the blood sample collection. Figure 1 was created using BioRender.com.

\section{Authors' contributions}

SP and MV contributed to the development and maintenance of the Doetinchem Cohort Study. LS, PE, AB, and MB developed and implemented the DCS subcohort study design. MZ and LS developed the flow cytometry laboratory protocols and $\mathrm{MZ}$ performed the experimental work in the laboratory and performed initial data cleaning. JF provided the initial data analysis framework, and LS and PE implemented it in the manuscript. LS performed data cleaning, wrote the initial draft of the manuscript, and provided visualizations. All authors read and approved the final manuscript.

\section{Funding}

The Doetinchem Cohort Study is funded by the Dutch Ministry of Health, Welfare and Sport. Additional funding for the current study was also provided by the Ministry. The funder had no role in study design, data collection and analysis, decision to publish, or preparation of the manuscript.

\section{Availability of data and materials}

The datasets generated and/or analyzed during the current study are not publicly available due to privacy and ethical restrictions but are available from the corresponding author on reasonable request.

\section{Declarations}

Ethics approval and consent to participate

The study was approved by the Medical Ethics Committee of the University Medical Center in Utrecht, The Netherlands. The participants gave written informed consent for every DCS round and for this subcohort study separately.

\section{Consent for publication}

Not applicable.

\section{Competing interests}

None to declare.

\section{Author details}

${ }^{1}$ National Institute of Public Health and the Environment, Bilthoven, The Netherlands. ${ }^{2}$ Department of Rheumatology and Clinical Immunology, University of Groningen, University Medical Center Groningen, Groningen, The Netherlands. ${ }^{3} J u l i u s$ Center for Health Sciences and Primary Care, University Medical Center Utrecht, Utrecht University, Utrecht, The Netherlands.

Received: 15 October 2021 Accepted: 23 December 2021 Published online: 17 January 2022

\section{References}

1. Kennedy BK, Berger SL, Brunet A, Campisi J, Cuervo AM, Epel ES, et al. Geroscience: linking aging to chronic disease. Cell. 2014;159(4):709-13.

2. Glynn JR, Moss PAH. Systematic analysis of infectious disease outcomes by age shows lowest severity in school-age children. Sci Data. 2020;7(1):329. 
3. Goodwin K, Viboud C, Simonsen L. Antibody response to influenza vaccination in the elderly: a quantitative review. Vaccine. 2006;24:1159.

4. Gustafson CE, Kim C, Weyand CM, Goronzy JJ. Influence of immune aging on vaccine responses. J Allergy Clin Immunol. 2020;145(5):1309-21.

5. Aiello A, Farzaneh F, Candore G, Caruso C, Davinelli S, Gambino CM, et al. Immunosenescence and its hallmarks: how to oppose aging strategically? A review of potential options for therapeutic intervention. Front Immunol. 2019:10:2247.

6. Franceschi $\mathrm{C}$, Bonafè $\mathrm{M}$, Valensin $\mathrm{S}$, Olivieri $\mathrm{F}$, De Luca $\mathrm{M}$, Ottaviani $\mathrm{E}$, et al. Inflamm-aging: an evolutionary perspective on Immunosenescence. Ann N Y Acad Sci. 2006;908(1):244-54

7. Fulop T, Witkowski JM, Olivieri F, Larbi A. The integration of inflammaging in age-related diseases. Semin Immunol. 2018;40:17-35.

8. Furman D, Campisi J, Verdin E, Carrera-Bastos P, Targ S, Franceschi C, et al. Chronic inflammation in the etiology of disease across the life span. Nat Med. 2019;25(12):1822-32.

9. Franceschi C, Garagnani P, Vitale G, Capri M, Salvioli S. Inflammaging and 'Garb-aging'. Trends Endocrinol Metab. 2017;28(3):199-212.

10. van Deursen JM. The role of senescent cells in ageing. Nature. 2014 509(7501):439-46.

11. Villarino AV, Kanno Y, O'Shea JJ. Mechanisms and consequences of Jak-STAT signaling in the immune system. Nat Immunol. 2017;18(4): 374-84.

12. O'Shea JJ, Schwartz DM, Villarino AV, Gadina M, McInnes IB, Laurence A. The JAK-STAT pathway: impact on human disease and therapeutic intervention. Annu Rev Med. 2015;66:311-28.

13. Dupuis S, Jouanguy E, Al-Hajjar S, Fieschi C, Al-Mohsen IZ, Al-Jumaah $\mathrm{S}$, et al. Impaired response to interferon- $\alpha / \beta$ and lethal viral disease in human STAT1 deficiency. Nat Genet. 2003;33(3):388-91.

14. Sancho-Shimizu V, Perez de Diego R, Jouanguy E, Zhang S-Y, Casanova J-L. Inborn errors of anti-viral interferon immunity in humans. Curr Opin Virol. 2011;1(6):487-96.

15. Sandborn WJ, Su C, Sands BE, D'Haens GR, Vermeire S, Schreiber S, et al. Tofacitinib as induction and maintenance therapy for ulcerative colitis. N Engl J Med. 2017;376(18):1723-36.

16. van der Heijde D, Strand V, Tanaka Y, Keystone E, Kremer J, Zerbini CAF, et al. Tofacitinib in combination with methotrexate in patients with rheumatoid arthritis: clinical efficacy, radiographic, and safety outcomes from a twentyfour-month, phase III study. Arthritis Rheum. 2019;71(6):878-91.

17. Shen-Orr SS, Furman D, Kidd BA, Hadad F, Lovelace P, Huang YW, et al. Defective signaling in the JAK-STAT pathway tracks with chronic inflammation and cardiovascular risk in aging humans. Cell Syst. 2016;3(4): 374-384 e4.

18. Piber D, Olmstead R, Cho JH-J, Witarama T, Perez C, Dietz N, et al. Inflammaging: age and systemic, cellular, and nuclear inflammatory biology in older adults. Le Couteur D, editor. J Gerontol Ser A. 2019;74(11):1716-24.

19. Febvre-James $M$, Lecureur $V$, Fardel O. Potent repression of C-reactive protein (CRP) expression by the JAK1/2 inhibitor ruxolitinib in inflammatory human hepatocytes. Inflamm Res. 2020;69(1):51-62.

20. Xu M, Tchkonia T, Ding H, Ogrodnik M, Lubbers ER, Pirtskhalava T, et al. JAK inhibition alleviates the cellular senescence-associated secretory phenotype and frailty in old age. Proc Natl Acad Sci U S A. 2015;112(46):E6301-10.

21. Bailey KL, Smith LM, Heires AJ, Katafiasz DM, Romberger DJ, LeVan TD. Aging leads to dysfunctional innate immune responses to TLR2 and TLR4 agonists. Aging Clin Exp Res. 2019:31(9):1185-93.

22. van den Biggelaar AHJ, Huizinga TWJ, de Craen AJM, Gussekloo J, Heijmans $B T$, Frölich M, et al. Impaired innate immunity predicts frailty in old age. The Leiden 85-plus study. Exp Gerontol. 2004;39(9):1407-14.

23. Wang Q, Westra J, van der Geest KSM, Moser J, Biizet J, Kuiper T, et al. Reduced levels of cytosolic DNA sensor AIM2 are associated with impaired cytokine responses in healthy elderly. Exp Gerontol. 2016;78:39-46.

24. Mitnitski AB, Mogilner AJ, Rockwood K. Accumulation of deficits as a proxy measure of aging. TheScientificWorldJournal. 2001;1:323-36.

25. Searle SD, Mitnitski A, Gahbauer EA, Gill TM, Rockwood K. A standard procedure for creating a frailty index. BMC Geriatr. 2008;8:24.

26. Picavet HSJ, Blokstra A, Spijkerman AMW, Verschuren WMM. Cohort profile update: the Doetinchem cohort study 1987-2017: lifestyle, health and chronic diseases in a life course and ageing perspective. Int J Epidemiol. 2017;46(6):1751-1751g.

27. Verschuren WM, Blokstra A, Picavet HS, Smit HA. Cohort profile: the Doetinchem cohort study. Int J Epidemiol. 2008;37(6):1236-41.
28. Van der Sluis RM, Zerbato JM, Rhodes JW, Pascoe RD, Solomon A, Kumar $N A$, et al. Diverse effects of interferon alpha on the establishment and reversal of HIV latency. Douek DC, editor. PLoS Pathog. 2020;16(2):e1008151.

29. Tsai WL, Vian L, Giudice V, Kieltyka J, Liu C, Fonseca V, et al. High throughput PSTAT signaling profiling by fluorescent cell barcoding and computational analysis. J Immunol Methods. 2020;477:112667.

30. Twohig JP, Cardus Figueras A, Andrews R, Wiede F, Cossins BC, Derrac Soria $A$, et al. Activation of naïve CD4+ T cells re-tunes STAT1 signaling to deliver unique cytokine responses in memory CD4+ T cells. Nat Immunol. 2019; 20(4):458-70.

31. Samson LD, Boots AMH, Ferreira JA, Picavet HSJ, de Rond LGH, de ZeeuwBrouwer $\mathrm{M}$, et al. In-depth immune cellular profiling reveals sex-specific associations with frailty. Immun Ageing. 2020;17(1):20.

32. Marcos-Pérez D, Sánchez-Flores M, Maseda A, Lorenzo-López L, MillánCalenti JC, Gostner JM, et al. Frailty in older adults is associated with plasma concentrations of inflammatory mediators but not with lymphocyte subpopulations. Front Immunol. 2018;9:1056.

33. Samson $L D, B$ Bots $A M H$, Verschuren WMM, Picavet HSJ, Engelfriet $P$, Buisman A-M. Frailty is associated with elevated CRP trajectories and higher numbers of neutrophils and monocytes. Exp Gerontol. 2019;125:110674.

34. Walker KA, Walston J, Gottesman RF, Kucharska-Newton A, Palta P, Windham BG. Midlife systemic inflammation is associated with frailty in later life: the ARIC study. J Gerontol A Biol Sci Med Sci. 2018;34(3):343-349.

35. Han H, Ma Q, Li C, Liu R, Zhao L, Wang W, et al. Profiling serum cytokines in COVID-19 patients reveals IL-6 and IL-10 are disease severity predictors. Emerg Microbes Infect. 2020;9(1):1123-30.

36. Lu L, Zhang H, Dauphars DJ, He Y-W. A potential role of interleukin 10 in COVID-19 pathogenesis. Trends Immunol. 2020; Available from: http://www. sciencedirect.com/science/article/pii/S1471490620302568. [cited 2020 Dec 22].

37. Takahashi T, Ellingson MK, Wong P, Israelow B, Lucas C, Klein J, et al. Sex differences in immune responses that underlie COVID-19 disease outcomes. Nature. 2020;588(7837):315-20.

38. Luo W, Li Y-X, Jiang L-J, Chen Q, Wang T, Ye D-W. Targeting JAK-STAT signaling to control cytokine release syndrome in COVID-19. Trends Pharmacol Sci. 2020;41(8):531-43.

39. Hojyo S, Uchida M, Tanaka K, Hasebe R, Tanaka Y, Murakami M, et al. How COVD-19 induces cytokine storm with high mortality. Inflamm Regen. 2020;40(1):37.

40. Gozzetti A, Capochiani E, Bocchia M. The Janus kinase 1/2 inhibitor ruxolitinib in COVID-19. Leukemia. 2020;34(10):2815-6.

41. La Rosée F, Bremer HC, Gehrke I, Kehr A, Hochhaus A, Birndt S, et al. The Janus kinase 1/2 inhibitor ruxolitinib in COVID-19 with severe systemic hyperinflammation. Leukemia. 2020;34(7):1805-15.

42. Kalil AC, Patterson TF, Mehta AK, Tomashek KM, Wolfe CR, Ghazaryan V, et al. Baricitinib plus Remdesivir for hospitalized adults with Covid-19. N Engl J Med. 2020; Available from: https://www.nejm.org/doi/10.1056/ NEJMoa2031994. [cited 2020 Dec 22].

43. Novartis. Novartis provides update on RUXCOVID study of ruxolitinib for hospitalized patients with COVID-19. Novartis. 2020; Available from: https:// www.novartis.com/news/media-releases/novartis-provides-update-ruxcovidstudy-ruxolitinib-hospitalized-patients-covid-19. [cited 2020 Dec 23].

44. Dodington DW, Desai HR, Woo M. JAK/STAT - emerging players in metabolism. Trends Endocrinol Metab. 2018;29(1):55-65.

45. Sehgal PB, Yang Y-M, Yuan H, Miller EJ. STAT5a/b contribute to sex bias in vascular disease: a neuroendocrine perspective. JAK-STAT. 2015;4(3):1-20.

46. Liao Z, Lutz J, Nevalainen MT. Transcription factor Stat5a/b as a therapeutic target protein for prostate cancer. Int J Biochem Cell Biol. 2010;42(2):186-92.

47. Leehy KA, Truong TH, Mauro L, Lange CA. Progesterone receptors (PR) mediate STAT actions: PR and prolactin receptor signaling crosstalk in breast cancer models. J Steroid Biochem Mol Biol. 2018;176:88-93.

48. Young NA, Valiente GR, Hampton JM, Wu L-C, Burd CJ, Willis WL, et al. Estrogen-regulated STAT1 activation promotes TLR8 expression to facilitate signaling via microRNA-21 in systemic lupus erythematosus. Clin Immunol. 2017:176:12-22

49. Duong L, Radley H, Lee B, Dye D, Pixley F, Grounds M, et al. Macrophage function in the elderly and impact on injury repair and cancer. Immun Ageing. 2021;18(1):4.

50. Samson LD, Buisman A-M, Ferreira JA, Picavet HSJ, Verschuren WMM, Boots $\mathrm{AMH}$, et al. Relation of inflammatory marker trajectories with frailty and aging in a 20-year longitudinal study. bioRxiv. 2021:2021.02.10.430670. https://doi.org/10.1101/2021.02.10.430670. 
51. Collerton J, Martin-Ruiz C, Davies K, Hilkens CM, Isaacs J, Kolenda C, et al. Frailty and the role of inflammation, immunosenescence and cellular ageing in the very old: cross-sectional findings from the Newcastle $85+$ study. Mech Ageing Dev. 2012;133(6):456-66.

52. Schoufour JD, Erler NS, Jaspers L, Kiefte-de Jong JC, Voortman T, Ziere G, et al. Design of a frailty index among community living middle-aged and older people: the Rotterdam study. Maturitas. 2017;97:14-20.

53. Krutzik PO, Nolan GP. Fluorescent cell barcoding in flow cytometry allows high-throughput drug screening and signaling profiling. Nat Methods. 2006; 3(5):361-8.

54. Hulsegge G, Herber-Gast GC, Spijkerman AM, Susan H, Picavet J, van der Schouw YT, et al. Obesity and age-related changes in markers of oxidative stress and inflammation across four generations. Obesity (Silver Spring). 2016;24(6):1389-96.

55. Hothorn $T$, Winell H, Hornik K, van de Wiel MA, Zeileis A. Coin: conditional inference procedures in a permutation test framework. 2019. Available from: https://CRAN.R-project.org/package=coin.

56. Benjamini $Y$, Hochberg Y. Controlling the false discovery rate: a practical and powerful approach to multiple testing. J R Stat Soc Ser B Methodol. 1995;57(1):289-300.

57. R Core Team. R: a language and environment for statistical computing. Vienna: R Foundation for Statistical Computing; 2019. Available from: https://www.R-project.org/

58. Wickham H, François R, Henry L, Müller K. dplyr: a grammar of data manipulation. 2020. Available from: https://CRAN.R-project.org/package=dplyr.

59. Wickham H, Henry L. tidyr: tidy messy data. 2020. Available from: https:// CRAN.R-project.org/package=tidyr.

60. Gu Z, Eils R, Schlesner M. Complex heatmaps reveal patterns and correlations in multidimensional genomic data. Bioinformatics. 2016;32(18):2847-9.

61. Wickham H, Chang W, Henry L, Pedersen TL, Takahashi K, Wilke C, et al. ggplot2: create elegant data visualisations using the grammar of graphics. 2019. Available from: https://CRAN.R-project.org/package=ggplot2.

62. Wilke CO. cowplot: Streamlined plot theme and plot annotations for "ggplot2". 2019. Available from: https://CRAN.R-project.org/package=cowplot.

63. Xie Y. knitr: a general-purpose package for dynamic report generation in $\mathrm{r}$. 2020. Available from: https://yihui.org/knitr/.

64. Zhu H. kableExtra: construct complex table with "kable" and pipe syntax. 2020. Available from: https://CRAN.R-project.org/package=kableExtra.

\section{Publisher's Note}

Springer Nature remains neutral with regard to jurisdictional claims in published maps and institutional affiliations.

Ready to submit your research? Choose BMC and benefit from:

- fast, convenient online submission

- thorough peer review by experienced researchers in your field

- rapid publication on acceptance

- support for research data, including large and complex data types

- gold Open Access which fosters wider collaboration and increased citations

- maximum visibility for your research: over $100 \mathrm{M}$ website views per year

At $\mathrm{BMC}$, research is always in progress.

Learn more biomedcentral.com/submissions 